\title{
Impacto económico de la introducción de la vacuna inactivada inyectable contra la poliomielitis en Colombia
}

\author{
Nelson Alvis, ${ }^{1}$ Fernando De la Hoz ${ }^{2}$ y Javier Narváez ${ }^{2}$
}

Forma de citar

Alvis N, De la Hoz F, Narváez J. Impacto económico de la introducción de la vacuna inactivada inyectable contra la poliomielitis en Colombia. Rev Panam Salud Publica. 2010;27(5):352-9.

RESUMEN Objetivo. Evaluar la relación costo-efectividad de la introducción de la vacuna inyectable contra la poliomielitis (VIP) en Colombia con respecto al sistema actual basado en el empleo de la vacuna oral (VOP).

Métodos. Se diseñó un modelo de Markov basado en una cohorte hipotética de recién nacidos que recibiría la VIP o la VOP con un seguimiento de dos años y estimaciones mensuales del número de casos de poliomielitis paralítica asociada con la vacuna (PPAV). El análisis del costo se realizó desde la perspectiva del asegurador (costos a lo largo de la vida) y la sociedad (casos de PPAV evitados y años de vida ajustados por discapacidad [AVAD] evitados).

Resultados. Entre 1988 y 1998 se aplicaron en Colombia 22,5 millones de dosis de la VOP y se detectaron nueve casos de PPAV, para una tasa de $4,0 \times 10^{-7}$ por dosis. Según el modelo, se podrían esperar entre 2 y 4 casos de PPAV en los dos años de seguimiento. El costo de tratar los casos de PPAV sería de US\$ 302 008, con costos de vacunación con la VOP de US\$ 737037 y de US\$ 5527777 con la VIP. La vacunación con la VIP permitiría evitar 64 $A V A D$ con un costo de US\$ 71062 por AVAD evitado; evitar un caso de PPAV mediante la sustitución de la VOP por la VIP costaría entre US\$1,8 millones y US\$2,2 millones.

Conclusiones. La sustitución de la VOP por la VIP no es una medida efectiva en función del costo en Colombia, incluso si se sustituyera la vacuna celular contra la tos ferina, actualmente en uso, por una vacuna acelular combinada con una VIP.

Palabras clave Vacuna antipolio oral; vacuna antipolio de virus inactivados; análisis costo-beneficio; años de vida perdidos por incapacidad; vacunación masiva; Colombia.

Medidas internacionales como la Iniciativa de Erradicación Mundial de la Poliomielitis (GPEI) han contribuido a

\footnotetext{
1 Grupo de Investigación en Economía de la Salud, Facultad de Ciencias Económicas, Universidad de Cartagena, Colombia. La correspondencia se debe dirigir a Nelson Alvis, Facultad de Ciencias Económicas, Universidad de Cartagena, Campus de la Piedra de Bolívar, Cartagena, Colombia. Correo electrónico: nalvis@yahoo.com, nalvis@gmail.com

2 Facultad de Medicina, Universidad Nacional de Colombia, Bogotá, D.C., Colombia.
}

reducir en 125 países donde esta enfermedad es endémica el número de casos registrados de 350000 en 1988 a solo 680 en 2006 (1-3). En la actualidad, el virus de la poliomielitis sigue siendo endémico en solo seis países: Afganistán, India, Nigeria, Níger, Egipto y Pakistán (4). A pesar de algunos reveses, el esfuerzo por erradicarlo no se ha detenido $\mathrm{y}$, con el ímpetu ganado con el uso de la vacuna covalente oral contra la poliomielitis, la Organización Mundial de la
Salud (OMS) anticipa que la transmisión de este virus se interrumpirá a finales de la década de 2010 (4).

Varios autores han estimado el efecto económico de la erradicación de la poliomielitis en el mundo (5-8). En los Estados Unidos de América, por ejemplo, además de evitar cientos de miles de casos de poliomielitis paralítica y muertes prematuras, los beneficios económicos netos en dólares estadounidenses (US\$) de los programas de vacunación 
excedieron los US\$ 180000 millones, sin contar los beneficios intangibles relacionados con el temor y el sufrimiento de las familias (9). Estos beneficios exceden la inversión acumulada en casi 20 años -con una mayor contribución nacional— de más de US\$ 4000 millones (8).

Con la eliminación de la circulación del virus natural de la poliomielitis en América Latina, incluida Colombia, la aparición de casos ocasionales asociados con el virus vacunal se ha convertido en un tema de debate de salud pública. Una de las alternativas para reducir este problema es el cambio de la vacuna oral a la vacuna inactivada inyectable de Salk, que además tiene una mayor inmunogenicidad y se puede combinar con otras vacunas. Sin embargo, según un documento de la OMS, la introducción de la vacuna inyectable contra la poliomielitis (VIP) en los países tropicales podría enfrentar varios retos importantes relacionados con el desconocimiento de algunos aspectos fundamentales, como los posibles altos costos de su introducción $(10,11)$, su inmunogenicidad en las condiciones específicas de esos países aunque se aplique según los esquemas recomendados y el efecto que tendría en la respuesta humoral de la mucosa intestinal ante la circulación de virus derivados de la vacuna oral contra la poliomielitis (VOP). Se ha calculado que pasar en los Estados Unidos a un esquema de vacunación basado en la VIP - ya sea sola o aplicada en dos dosis seguidas de dos dosis de la VOP - costaría US\$ 28,1 millones o US\$ 14,7 millones, según la opción escogida, y el costo por caso de poliomielitis evitado sería de US\$ 3,0 millones o US\$ 3,1 millones, respectivamente; aunque estos valores podrían disminuir notablemente si se redujera el número de inyecciones por la aplicación de vacunas combinadas (12).

Las recomendaciones de la OMS para sustituir la VOP por la VIP en los países que se consideran en riesgo varían en dependencia de la decisión de los países de mantener el virus natural de la poliomielitis en sus laboratorios de vigilancia. En los que decidan conservar muestras de virus natural colectadas durante la vigilancia epidemiológica antes de su eliminación, la recomendación es mantener altos niveles de inmunidad poblacional mediante la aplicación de la VIP a las 14 semanas de edad con una segunda dosis 6 meses después. Una alternativa sería aplicar de 3 a 4 dosis de la VIP a partir de las 6 semanas de edad con intervalos de 4 a 8 semanas. Los países que no se perciben en riesgo pueden suspender la vacunación sistemática contra la poliomielitis, mantener y fortalecer la vigilancia de los casos de esta enfermedad - ya sea por el virus natural o el vacunal-, elevar la capacidad de respuesta para controlar un posible brote de poliomielitis y apoyarse en la OMS si se necesita la VOP para controlar alguna emergencia $(10,13)$.

En el presente estudio se evalúa la relación costo-efectividad de la introducción de la VIP en el Programa Ampliado de Inmunizaciones (PAI) de Colombia con respecto al sistema actual basado en el empleo de la VOP.

\section{MATERIALES Y MÉTODOS}

El análisis de la relación costo-efectividad se apoyó en una revisión de los artículos publicados en revistas científicas colombianas y extranjeras sobre la VIP, indizadas en las bases de datos bibliográficas Medline, SciELO y LILACS, con los términos de búsqueda "vaccine-associated paralytic poliomyelitis", "inactivated poliovirus vaccine AND prevalence OR incidence OR epidemiology", así como sus equivalentes en español. En esta revisión bibliográfica se buscaron los artículos publicados entre 1998 y 2008 que presentaran datos originales sobre uno o varios de los siguientes aspectos: a) proceso de introducción de la VIP, ya sea en países desarrollados o en desarrollo; b) análisis de la relación costo-efectividad de la VIP en cualquiera de sus formas de presentación; c) análisis de efectividad o de eficacia de la VIP; y d) análisis de los efectos secundarios, tanto de la VOP como de la VIP. El objetivo de esta búsqueda fue identificar indicadores de ocurrencia de casos de parálisis asociada con la VOP, estimadores de costos de la poliomielitis paralítica -independientemente de su asociación con la VOP - y estimaciones del costo en función de la efectividad del reemplazo de la VOP por otras estrategias.

A partir de los resúmenes de los artículos recuperados se seleccionaron los que contenían información sobre la incidencia, la prevalencia o la mortalidad en Colombia o en otros países de América Latina y se analizó el texto completo. Se excluyeron del análisis los artículos centrados en aspectos biomédicos o moleculares de las vacunas.

\section{Fuentes de los datos}

El estudio de costos de la enfermedad se realizó desde las perspectivas del asegurador $^{3}$ y la sociedad a partir de dos elementos: los costos por caso informados en los registros individuales de prestación de servicios (RIPS) del Ministerio de la Protección Social de Colombia y el ajuste de esos costos según el consenso de expertos. Para el cálculo del costo a lo largo de la vida se tomaron los costos recurrentes de la atención de los casos de poliomielitis paralítica asociada con la vacuna (PPAV). Los costos se calcularon en pesos colombianos (\$) y se convirtieron a dólares estadounidenses del año 2008, con una tasa de cambio de US\$1,00 = \$2 234 (14).

Los datos epidemiológicos se tomaron de los resultados de la vigilancia virológica que se realiza en Colombia sobre los casos de parálisis flácida aguda, coordinada por el Laboratorio de Virología del Instituto Nacional de Salud de Colombia (15).

Los datos de la estructura y la dinámica de la población colombiana se tomaron de las proyecciones de población del Departamento Administrativo Nacional de Estadísticas (16).

Los costos de la vacunación abarcaron varios componentes: a) el precio de las dosis; b) los costos asociados con la transportación, la importación, la administración del programa de vacunación y la merma; y c) los costos del programa, asociados con el entrenamiento del personal, la movilización social, la transportación dentro del país, el seguimiento y la vigilancia epidemiológica de los casos de PPAV, la cadena de frío, el mantenimiento del equipamiento y la gestión de los desechos. El precio de la VIP se obtuvo del Ministerio de la Protección Social (cuadro 1).

Para los cálculos se consideró que la eficacia de la VIP en evitar casos de PPAV era de $100 \%$ después de tres dosis y de 93\% después de dos dosis (17). Como la VIP se aplicaría conjuntamente con la vacuna combinada contra la difteria, el tétanos, la tos ferina, la influenza tipo b y la hepatitis B, se consideró una cobertura de $90 \%$, igual que la lograda con la vacuna

\footnotetext{
3 En el sistema de salud colombiano, las empresas promotoras brindan seguros de salud a la población por dos vías: un régimen contributivo que depende del ingreso de la persona según su actividad laboral y un régimen subsidiado para la población que no tiene capacidad de pago.
} 
CUADRO 1. Parámetros del modelo para evaluar la relación costo-efectividad de la sustitución de la vacuna inyectable contra la poliomielitis (VIP) con respecto a la vacuna oral (VOP) en el Programa Ampliado de Inmunizaciones de Colombia, 2008

\begin{tabular}{|c|c|c|c|c|}
\hline Parámetro & Dato base & Límite inferior & Límite superior & Referencia \\
\hline Número de nacimientos & 818930 & 777984 & 859877 & 16 \\
\hline Dosis de la VOP aplicadas en 2007 & 2334000 & 2217300 & 2450700 & 17 \\
\hline Casos de PPAVa por dosis aplicada (1980-1989) & $1,0 \times 10^{-6}$ & $7,1 \times 10^{-7}$ & $1,63 \times 10^{-6}$ & $18,19,20$ \\
\hline Costo de la VOPb & 1,5 & 1,0 & 3,0 & $\mathrm{PAI}^{\mathrm{c}}$ \\
\hline Costo de la VIP & 7,5 & 5,0 & 7,5 & $\mathrm{PAl}^{\mathrm{C}}$ \\
\hline Costo anual de tratar un caso de PPAVb & 5000 & 2500 & 7500 & 18 \\
\hline Tasa de descuento anual (\%) & 3,0 & $N A^{d}$ & NA & NA \\
\hline
\end{tabular}

a PPAV: poliomielitis paralítica asociada con la vacuna.

${ }^{\mathrm{b}}$ En dólares estadounidenses (US\$) de 2008; tasa de cambio por peso colombiano (\$): US\$1,00 = \$2 234 (14).

c Dra. Martha Velandia, coordinadora nacional del PAI en Colombia, comunicación personal.

${ }^{\mathrm{N}} \mathrm{NA}$ : no aplica.

pentavalente en Colombia. Para la modelación se tomó también una cobertura de vacunación de $90 \%$ para la VOP.

\section{Descripción del modelo}

Para el análisis de la relación costoefectividad se diseñó un modelo de Markov (figura 1) basado en una cohorte hipotética de recién nacidos que recibiría la VIP o la VOP. Esta cohorte tuvo un seguimiento de dos años, con estimaciones mensuales del número de casos de PPAV por cada dosis recibida, según el esquema habitual de vacunación. Las probabilidades estimadas de que un recién nacido de la cohorte alcance cada uno de los distintos estados de la enfermedad por los que podría transitar y los demás parámetros del modelo se ofrecen en el cuadro 1.

Como resultados finales del modelo se tomaron: a) casos de PPAV evitados; b) años de vida ajustados por discapacidad (AVAD) evitados; y c) costos a lo largo de la vida. El cálculo de los AVAD refleja la duración y el peso de la discapacidad estimada según el estudio de carga total de la enfermedad (21). Además se utilizó la razón costo-efectividad incremental (RCEI) para comparar las alternativas de vacunar o no vacunar, mediante la siguiente fórmula:

(Costos de la vacunación con la VIP RCEI $=\frac{\text { Costos de casos de PPAV evitados) }}{\text { AVAD evitados por la vacunación }}$

Se consideró que con una RCEI superior a tres veces el producto interno bruto per cápita de Colombia, la introducción de la VIP no resultaría efectiva en función del costo, mientras que si estaba por debajo de ese valor sería efectiva (22). Además,
FIGURA 1. Modelo de Markov para valorar la relación costo-efectividad de la vacuna inyectable contra la poliomielitis (VIP) con respecto a la vacuna oral (VOP) en Colombia, 2008

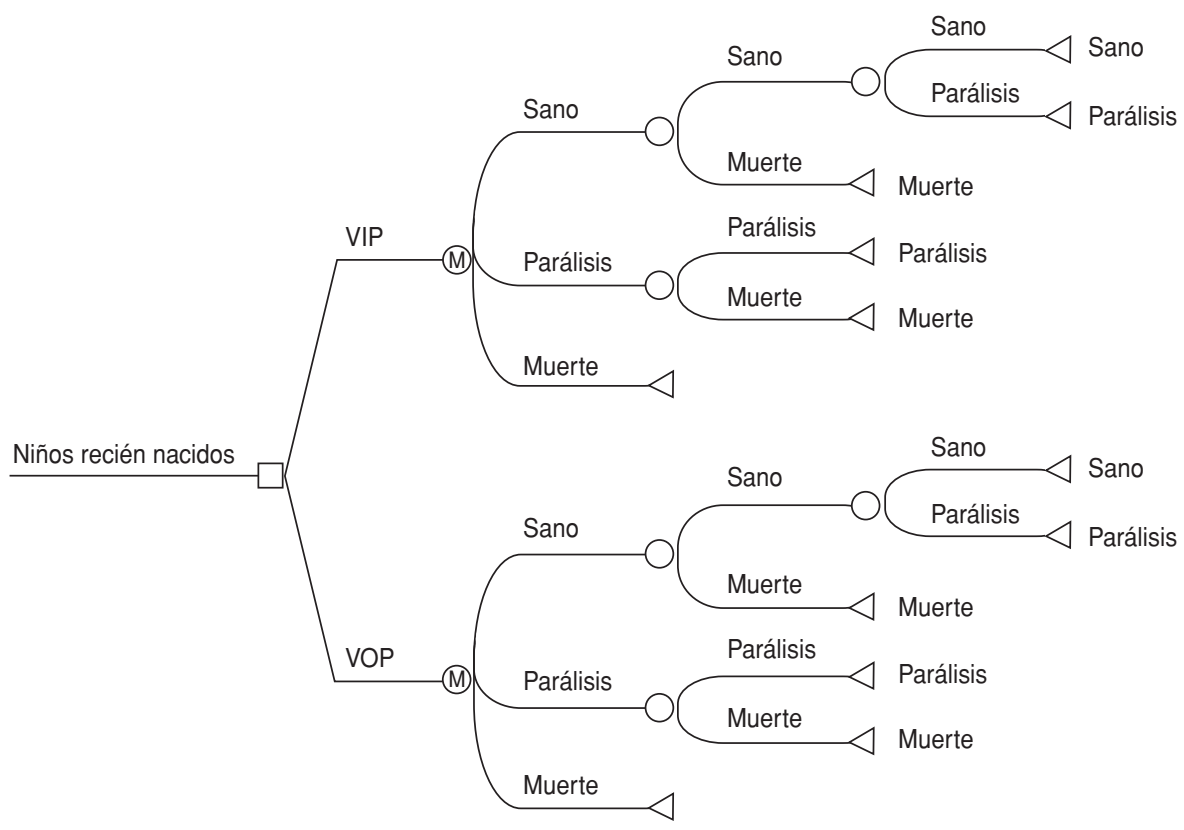

se consideró que $5 \%$ de los casos de PPAV morirían $(23,24)$ y que la parálisis flácida aguda aparece a los 8 meses de edad y permanece por el resto de la vida, con una esperanza de vida de 75 años, similar a la del resto de la población (23). El peso de la discapacidad para los sobrevivientes, con tratamiento o no, se fijó en 0,369 en una escala de estado de salud de 0 a 1 ( 0 : mejor; 1 : peor) (21). Para valorar tanto los costos como los resultados del seguimiento, se utilizó una tasa de descuento anual de $3 \%$.

\section{Análisis de sensibilidad}

Para el análisis de sensibilidad se aplicaron, en un diagrama de tornado, valores límites inferiores y superiores de los indicadores estudiados (cuadro 1) y de $85 \%$ y $95 \%$, respectivamente, para la cobertura de vacunación. En este método, cada variable se somete secuencialmente al análisis de sensibilidad de una vía para establecer un ordenamiento de las mismas según su influencia en la variación de los resultados. Posteriormente se realizó un análisis de sensibilidad determinístico, en el que se emplearon las variables identificadas con mayor efecto económico y epidemiológico sobre los resultados.

\section{RESULTADOS}

No se encontraron estudios relacionados con la incidencia o el análisis de 
CUADRO 2. Riesgo estimado de poliomielitis paralítica asociada con la vacuna oral en diversos países y regiones, según estudios revisados

\begin{tabular}{|c|c|c|c|c|c|}
\hline País/región & $\begin{array}{l}\text { Período de } \\
\text { observación }\end{array}$ & $\begin{array}{l}\text { Millones de } \\
\text { primeras dosis }\end{array}$ & $\begin{array}{l}\text { Riesgo } \\
\text { por dosis }\end{array}$ & Otros datos & Referencia \\
\hline América Latina & 1989 y 1991 & 1,2 & $8,3 \times 10^{-7}$ & $\begin{array}{l}\text { Los serotipos } 1,3 \text { y } 2 \text { fueron los más frecuentes }(38,33 \text { y } 24 \\
\text { casos, respectivamente). En ese mismo período, Colombia } \\
\text { aportaba } 12 \text { casos al hallazgo regional. El riesgo fue de } 2,7 \times 10^{-7} \\
\text { después de } 3,6 \text { millones de dosis totales. }\end{array}$ & 25 \\
\hline Brasil & 1989-1995 & 2,4 & $4,1 \times 10^{-7}$ & $\begin{array}{l}\text { El riesgo fue de } 8,0 \times 10^{-8} \text { después de } 13 \text { millones de dosis } \\
\text { totales. Los serotipos de poliomielitis } 2 \text { y } 3 \text { fueron los más } \\
\text { frecuentemente hallados. }\end{array}$ & 26 \\
\hline Brasil & 1995-2001 & 5,0 & $2,0 \times 10^{-7}$ & $\begin{array}{l}\text { La mayoría de los casos se asociaron con el virus } 3 \text { ( } 6 \text { casos), } \\
\text { virus } 2 \text { ( } 5 \text { casos) y virus } 1 \text { ( } 4 \text { casos). Cuatro casos se asociaron } \\
\text { con dos serotipos. El riesgo fue de } 1,0 \times 10^{-7} \text { después de } \\
10 \text { millones de dosis totales. }\end{array}$ & 27 \\
\hline Cuba & 1963-2006 & 0,38 & $1,6 \times 10^{-6}$ & $\begin{array}{l}\text { Los niños de } 4-7 \text { meses de edad presentaron un riesgo mayor: } \\
7,0 \times 10^{-6} \text { después de } 132812 \text { primeras dosis. }\end{array}$ & 19 \\
\hline Estados Unidos & 1973-1984 & 3,2 & $3,1 \times 10^{-7}$ & & 28 \\
\hline Estados Unidos & 1980-1989 & 2,5 & $4,0 \times 10^{-7}$ & & 29 \\
\hline Inglaterra y Gales & 1985-1991 & 1,4 & $7,1 \times 10^{-7}$ & & 30 \\
\hline
\end{tabular}

casos de PPAV en Colombia, pero se encontraron siete estudios en los que se estimaba la incidencia de PPAV en otros países (cuadro 2).

Solo se encontraron tres estudios de costo-efectividad de la VIP. Griffiths y colaboradores estimaron que la RCEI de la introducción de la VIP en Sudáfrica estaría entre US\$ 740000 y US\$ 7,2 millones por caso de PPAV evitado, y el RCEI por AVAD evitado estaría entre US\$ 61000 y US\$ 594000 . A precios de la VIP entre US\$1,00 y US\$10,00, ninguna de las opciones analizadas resultó efectiva en función del costo en ese país (31). En Australia, Tucker y colaboradores evaluaron el impacto económico de introducir la VIP (monovalente o en combinación con otras vacunas) y encontraron que prevenir un caso de PPAV podía costar entre US\$17,0 millones y US\$ 43,9 millones, lo cual no resultaba efectivo en función del costo (32). Por otro lado, Miller y colaboradores encontraron que con la introducción de la VIP en los Estados Unidos, el costo de cada caso de PPAV evitado estaría entre US\$3,0 millones y US\$ 3,1 millones (12).

Entre 1988 y 1998 se aplicaron aproximadamente 22,5 millones de dosis de la VOP a menores de 5 años en Colombia, de ellas 8 millones correspondieron a primeras dosis. En ese período se detectaron nueve casos de PPAV, para una tasa general de $4,0 \times 10^{-7}$ por dosis aplicada y de $6,2 \times 10^{-7}$ por cada primera dosis. Cuatro casos se debían al serotipo 3, cuatro al serotipo 2 y uno al serotipo 1 . Tres de los nueve casos $(33,3 \%)$ procedían de Bogotá y $5(55,6 \%)$ presentaron los síntomas después de la primera dosis. Entre 1999 y 2005, se han confirmado virológicamente cuatro casos después de 10 millones de dosis aplicadas, de las cuales cerca de 3 millones eran primeras dosis.

Según el modelo elaborado para esta cohorte hipotética de recién nacidos vacunados con la VOP, se podrían esperar entre 2 y 4 casos de PPAV en los dos años de seguimiento. El costo de tratamiento de los casos de PPAV durante la vida, con el descuento anual previsto de 3\%, sería de US\$ 302008 y variaría entre US\$ 151004 y US\$ 453 011. Los costos de la vacunación con la VOP serían de US\$ 737037 , con límites entre US\$ 368519 y US\$ 1105 556, mientras que los costos de vacunación con la VIP serían de US\$ 5527 777, con posibles límites de US\$ 3685185 y US\$ 5527778.

En cuanto a los beneficios sobre la salud desde la perspectiva de la sociedad, la estrategia de vacunar con la VIP permitiría evitar 127 AVAD, que se reducirían a 64 AVAD si se toma en cuenta el descuento previsto. Esto representaría un costo de US\$ 32695 o US\$ 71062 por AVAD evitado, respectivamente (cuadro 3). Así, evitar un caso de PPAV mediante la sustitución de la VOP por la VIP costaría entre US\$1,8 millones y US\$ 2,2 millones (ajustados al valor de 2008).

\section{Análisis de sensibilidad}

El análisis de sensibilidad mostró que la variable que más influyó en la magnitud de los beneficios netos fue el costo de aplicar el esquema de vacunación con la VOP, que explicaría cerca de $79 \%$ de la variación observada en el diagrama de tornado, seguida del costo del tratamiento de la PPAV (figura 2). Según el análisis de sensibilidad determinístico de una vía —aplicado por separado a los costos de VOP y de VIP-, si el precio de la dosis de la VOP fuera de US\$ 0,50, la RCEI sería de US\$ 76854 por AVAD evitado y si el costo de la VIP fuera de US\$ 5,00, la RCEI sería de US\$ 42098 por AVAD evitado (cuadro 4).

\section{DISCUSIÓN}

El presente estudio muestra que el costo de evitar un caso de PPAV en Colombia mediante el uso de la VIP estaría entre US\$ 1,8 millones y US\$2,2 millones y se gastarían entre US\$ 42000 y US\$ 77000 por cada AVAD evitado. Esto indica que la introducción de la VIP no es una estrategia efectiva en función del costo en este país, si se adopta el estándar de costo-efectividad fijado por la OMS (22). Estos resultados son similares a los obtenidos por Griffiths y colaboradores en 2006 en Sudáfrica (31), Miller y colaboradores en 1996 en los Estados Unidos (12) y Tucker y colaboradores en Australia (32).

En Colombia se han confirmado cuatro casos de PPAV después de 1999, a pesar de haberse aplicado más de 10 millones de dosis de la VOP, de las cuales 3 millones eran primeras dosis. Esta baja frecuencia es similar a la observada en Brasil $(26,27)$, donde la frecuencia de casos disminuyó 
CUADRO 3. Razón costo-efectividad incremental (RCEI) de la introducción de la vacuna inyectable contra la poliomielitis (VIP) con respecto a la vacuna oral contra la poliomielitis (VOP) en Colombia, 2008

\begin{tabular}{|c|c|c|c|c|c|}
\hline Estrategia & Costo (US\$) $)^{a}$ & $\begin{array}{c}\text { Costo } \\
\text { incremental } \\
(U S \$)^{a}\end{array}$ & 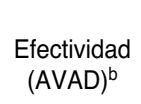 & $\begin{array}{c}\text { Efectividad } \\
\text { incremental } \\
\text { (AVAD) }\end{array}$ & $\begin{array}{c}\text { RCElc } \\
\text { (US\$/AVAD) }\end{array}$ \\
\hline \multicolumn{6}{|c|}{ Escenario sin descuento anual } \\
\hline VOP & 1375492 & 4152285 & 37495434 & 127 & 32695 \\
\hline VIP & 5527778 & & 37495562 & & \\
\hline \multicolumn{6}{|c|}{ Escenario con descuento de $3 \%$ anual } \\
\hline VOP & 1006974 & 4520804 & 18711165 & 64 & 71062 \\
\hline VIP & 5527778 & & 18711229 & & \\
\hline
\end{tabular}

a En dólares estadounidenses (US\$) de 2008; tasa de cambio por peso colombiano (\$): US\$1,00 = \$2 234 (14).

${ }^{b}$ La efectividad se midió en años de vida ajustados por discapacidad (AVAD) evitados.

${ }^{c}$ RCEI: razón costo-efectividad incremental, calculada como la razón entre el costo incremental y la efectividad incremental.

FIGURA 2. Diagrama de tornado para el análisis de sensibilidad de la relación costo-efectividad del uso de la vacuna inyectable contra la poliomielitis (VIP) con respecto a la vacuna oral (VOP) en el Programa Ampliado de Inmunizaciones de Colombia, 2008

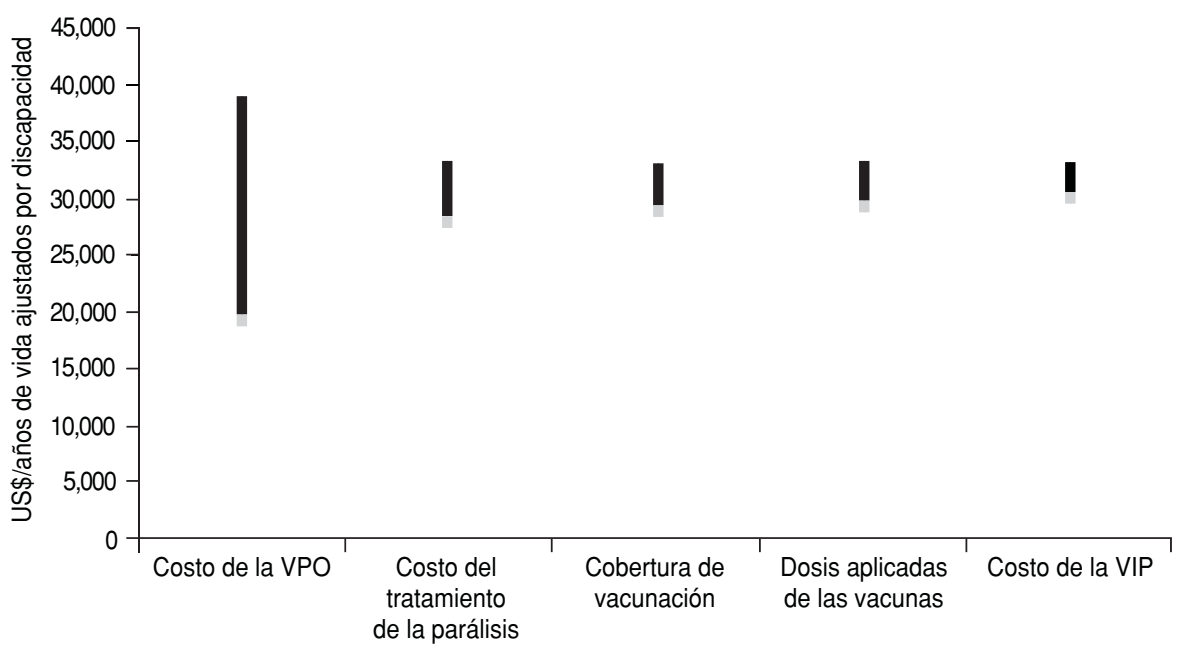

en más de la mitad entre 1995 y 2001 a pesar del uso continuado de la VOP. Sin embargo, no puede descartarse totalmente que la baja frecuencia de PPAV se deba a fallas de la vigilancia que lleven a subestimar la verdadera incidencia, ya que algunos indicadores nacionales de vigilancia de la poliomielitis - como el porcentaje de casos sospechados con muestra tomada en los primeros 14 días y el de unidades que notifican semanalmentehan sido más bajos en algunos municipios que el promedio de varios años en la región correspondiente $(33,34)$. El número de casos notificados en menores de 15 años de parálisis flácida aguda ha aumentado ligeramente entre 2005 y 2007 de menos de 1 por 100000 a más de 1,1 por 100 000; sin embargo, el nivel de notificación al parecer no es uniforme entre los 32 departamentos, pues en 11 de ellos la incidencia se encuentra por debajo de la tasa promedio nacional (34).
Se han estudiado los efectos de varias estrategias aplicables en los países libres de poliomielitis $(18,35)$ : a) suspender todo tipo de vacunación sistemática contra la poliomielitis y continuar solo con la vigilancia; b) sustituir la VOP por la VIP en un solo paso; c) cambiar parcialmente a la VIP, es decir remplazar las dosis de la VOP aplicadas a los 2 y 4 meses por dosis de VIP y mantener la administración de las dosis de la VOP a los 12-18 meses y 4-6 años; d) continuar con la VOP en el esquema habitual de vacunación contra la poliomielitis y suspender las vacunaciones suplementarias, por ejemplo en los llamados días nacionales de vacunación; y e) continuar la vacunación sistemática y las suplementarias con la VOP. En los Estados Unidos se eligió la tercera estrategia (c) en 1998, mientras que en el Reino Unido se adoptó la segunda (b) en 2006. La primera estrategia (a), aunque atractiva y efectiva en función del costo, no resulta factible aún, debido al riesgo de reintroducción del virus desde países en los que aún circula el virus natural de la poliomielitis. La estrategia más viable desde el punto de vista epidemiológico es continuar la vacunación sistemática contra la poliomielitis, aunque cada país debe decidir si continuará empleando la VOP o si la reemplaza por la VIP.

No existe una recomendación clara de la OMS para los países tropicales acerca de la conveniencia o no de sustituir la VOP por la VIP debido al aún limitado conocimiento sobre algunos aspectos importantes de esta última vacuna, ya expuestos más arriba (10). En el presente estudio se ofrecen los resultados del primer análisis económico de la introducción de la VIP en un país tropical de América Latina y se demuestra que esta medida no resulta favorable desde la perspectiva del asegurador. Desde la perspectiva de la sociedad, el ahorro por evitar la pérdida de productividad de los pacientes con PPAV ( 2 a 4 en la cohorte seguida) y los ingresos dejados de recibir durante los 45 años de vida económicamente activa - aún con descuento- estaría entre US\$ 120000 y US\$ 240000 (al valor de 2008). En el mejor de los escenarios (pleno empleo, costo de la dosis de VIP de US\$ 5,00 y 4 casos de PPAV), este ahorro representaría entre $3 \%$ y $6 \%$ de los costos de la vacunación con VIP, muy por debajo de los costos netos y la RCEI. De forma similar, los costos indirectos asociados con los casos de PPAV según el estudio realizado en Sudáfrica representaron entre $1,2 \%$ y $6,5 \%$ de los costos de la vacunación con la VIP (31).

Sin embargo, la decisión de reemplazar la VOP por la VIP no se puede basar solamente en la relación costo-efectividad, pues se deben tomar en cuenta 


\begin{tabular}{|c|c|c|c|c|c|c|}
\hline $\begin{array}{l}\text { Costo } \\
\text { de la } \\
\text { vacuna }\end{array}$ & $\begin{array}{c}\text { Vacuna a } \\
\text { emplear }\end{array}$ & $\begin{array}{l}\text { Costo } \\
\text { total } \\
(\text { US\$) }\end{array}$ & $\begin{array}{l}\text { Costo } \\
\text { incremental } \\
(\text { US } \$)^{\mathrm{a}}\end{array}$ & $\begin{array}{c}\text { Efectividad } \\
(\text { AVAD })^{\mathrm{b}}\end{array}$ & $\begin{array}{c}\text { Efectividad } \\
\text { incremental } \\
\text { (AVAD) }\end{array}$ & $\begin{array}{c}\text { RCEIc } \\
\text { (US\$/AVAD) }\end{array}$ \\
\hline \multicolumn{7}{|l|}{ VOP } \\
\hline \multirow[t]{2}{*}{0,50} & VOP & 638455 & 4889322 & 18711165 & 64 & 76854 \\
\hline & VIP & 5527778 & & 18711229 & & \\
\hline \multirow[t]{2}{*}{0,70} & VOP & 785863 & 4741915 & 18711165 & 64 & 74537 \\
\hline & VIP & 5527778 & & 18711229 & & \\
\hline \multirow[t]{2}{*}{0,90} & VOP & 933270 & 4594508 & 18711165 & 64 & 72220 \\
\hline & VIP & 5527778 & & 18711229 & & \\
\hline \multirow[t]{2}{*}{1,10} & VOP & 1080677 & 4447100 & 18711165 & 64 & 69903 \\
\hline & VIP & 5527778 & & 18711229 & & \\
\hline \multirow[t]{2}{*}{1,30} & VOP & 1228085 & 4299693 & 18711165 & 64 & 67586 \\
\hline & VIP & 5527778 & & 18711229 & & \\
\hline \multirow[t]{2}{*}{1,50} & VOP & 1375492 & 4152285 & 18711165 & 64 & 65269 \\
\hline & VIP & 5527778 & & 18711229 & & \\
\hline \multicolumn{7}{|l|}{ VIP } \\
\hline \multirow[t]{2}{*}{5,00} & VOP & 1006974 & 2678211 & 18711165 & 64 & 42098 \\
\hline & VIP & 3685185 & & 18711229 & & \\
\hline \multirow[t]{2}{*}{5,50} & VOP & 1006974 & 3046730 & 18711165 & 64 & 47891 \\
\hline & VIP & 4053704 & & 18711229 & & \\
\hline \multirow[t]{2}{*}{6,00} & VOP & 1006974 & 3415248 & 18711165 & 64 & 53684 \\
\hline & VIP & 4422222 & & 18711229 & & \\
\hline \multirow[t]{2}{*}{6,50} & VOP & 1006974 & 3783767 & 18711165 & 64 & 59476 \\
\hline & VIP & 4790741 & & 18711229 & & \\
\hline \multirow[t]{2}{*}{7,00} & VOP & 1006974 & 4152285 & 18711165 & 64 & 65269 \\
\hline & VIP & 5159259 & & 18711229 & & \\
\hline \multirow[t]{2}{*}{7,50} & VOP & 1006974 & 4520804 & 18711165 & 64 & 71062 \\
\hline & VIP & 5527778 & & 18711229 & & \\
\hline
\end{tabular}

otras consideraciones económicas, como el posible efecto en el presupuesto del PAI y su posible prioridad en comparación con otras medidas que actúen sobre otros problemas de salud pública de igual o mayor importancia.

En el presente estudio no se consideraron en toda su dimensión los posibles beneficios de cambiar la vacuna celular contra la tos ferina que se utiliza actualmente en Colombia por la vacuna acelular incluida en todas las vacunas combinadas actuales con la VIP. El principal beneficio de este cambio sería la disminución de algunos efectos secundarios sistémicos (convulsiones e hipotonía) asociados con el uso de la vacuna celular; sin embargo, este beneficio no ha sido comprobado en estudios controlados (36). Además, la vacuna acelular podría interferir con la respuesta inmunológica a otras vacunas, especialmente las que contienen el antígeno vacunal del Haemophilus influenzae tipo b polirribosil fosfato (37). Ekwueme y colaboradores compararon los beneficios económicos de ambos tipos de vacunas contra la tos ferina en los Estados Unidos y encontraron que ambas producían un efecto similar sobre la enfermedad y generaban ahorros netos para los servicios de salud, pero no se pudo demostrar que la vacuna acelular fuera económicamente más ventajosa que la celular (38).

Una posible limitación de este estudio es que la vigilancia de la parálisis flácida aguda en Colombia no ha satisfecho algunas metas, como la proporción de muestras tomadas oportunamente, lo cual puede haber llevado a subestimar la frecuencia de PPAV.

En conclusión, la sustitución de la VOP por la VIP no es una medida efectiva en función del costo en Colombia, un país tropical latinoamericano con ingresos medios. Aún si se tomara en cuenta el posible efecto benéfico de sustituir la vacuna celular contra la tos ferina, actualmente en uso, por una vacuna acelular combinada con una VIP no se modificaría significativamente la relación costo-beneficio encontrada. La decisión, entonces, debe basarse en consideraciones de tipo ético, económico (por el presupuesto del PAI) y de prioridades de la salud pública nacional.

Se debe fortalecer la vigilancia epidemiológica de la poliomielitis y mejorar el desempeño en algunos indicadores de la vigilancia - especialmente la proporción de casos investigados en menos de 48 horas y la proporción de casos con muestra obtenida en los primeros 14 días de la enfermedad- para mantener el país libre de poliomielitis por el virus natural en el futuro.

Agradecimientos. Esta investigación se realizó en su totalidad con financiamiento del Ministerio de la Protección Social de Colombia a través del Programa Ampliado de Inmunizaciones.

Declaración de conflictos de interés. Fernando De la Hoz ha recibido honorarios de Sanofi Pasteur por presentar conferencias sobre la carga de enfermedad por hepatitis A. Los demás autores declaran no tener ningún conflicto de interés. 


\section{REFERENCIAS}

1. Progress toward global poliomyelitis eradication, 2000. Morb Mortal Wkly Rep. 2001; 50(16):320-2, 331.

2. Global progress toward certifying polio eradication and laboratory containment of wild polioviruses. August 2002-August 2003. Morb Mortal Wkly Rep. 2003;52(47):1158-60.

3. Global Polio Eradication Initiative Strategic Plan, 2004. Morb Mortal Wkly Rep. 2004; 53(5):107-8.

4. Dutta A. Epidemiology of poliomyelitisoptions and update. Vaccine. 2008;26(45): 5767-73.

5. Bart KJ, Foulds J, Patriarca P. Global eradication of poliomyelitis: benefit-cost analysis. Bull World Health Organ. 1996;74(1):35-45.

6. Kahn MM, Ehreth J. Costs and benefits of polio eradication: along-run global perspective. Vaccine. 2003;21:702-5.

7. Musgrove P. Is polio eradication in the Americas economically justified? Bull Pan Am Health Organ. 1988;22(1):1-16.

8. Thompson KM, Tebbens RJ. Eradication versus control for poliomyelitis: an economic analysis. Lancet. 2007;369(9570):1363-71.

9. Thompson KM, Tebbens RJ. Retrospective cost-effectiveness analyses for polio vaccination in the United States. Risk Anal. 2006; 26(6):1423-40.

10. Introduction of inactivated poliovirus vaccine into oral poliovirus vaccine-using countries. Wkly Epidemiol Rec. 2003;78(28):241-52.

11. Kumate J. Cincuentenario de la vacuna Salk. Bol Med Hosp Infant Mex. 2005;62:239-41.

12. Miller MA, Sutter RW, Strebel PM, Hadler SC. Cost-effectiveness of incorporating inactivated poliovirus vaccine into the routine childhood immunization schedule. J Am Med Assoc. 1996;276(12):967-71.

13. Inactivated poliovirus vaccine following oral poliovirus vaccine cessation. Wkly Epidemiol Rec. 2006;81(15):137-44.

14. República de Colombia, Banco de la República. Series estadísticas. Tasas de cambio. Santa Fe de Bogotá: Banco de la República; 2009. Hallado en http://www.banrep.gov. co/series-estadisticas/see_ts_cam.htm. Acceso el 4 de marzo de 2010.

15. Boshell J, Peláez D, Camacho T, Castaño M, Bernal C. Programa de Erradicación de la Poliomielitis en Colombia: apoyo del laboratorio de virología, INS, 1992-1998. Biomédica. 1999; 19(1):175.

16. Departamento Administrativo Nacional de Estadísticas. Proyecciones de población. Hallado en http:/ / www.dane.gov.co/daneweb_ V09/index.php?option=com_content\&view= article\&id=75\&Itemid=72. Acceso el 16 de marzo de 2010.

17. República de Colombia, Ministerio de la Protección Social. Programa ampliado de inmunizaciones: coberturas de vacunación munici- pales 1998-2008. Santa Fe de Bogotá: Ministerio de Protección Social; sin año. Hallado en http:/ / www.minproteccionsocial. gov.co/pai/Gestión.aspx. Acceso el 16 de marzo de 2010.

18. Thompson KM, Tebbens RJ, Pallansch MA Kew OM, Sutter RW, Aylward RB, et al. The risks, costs, and benefits of possible future global policies for managing polioviruses. Am J Public Health. 2008;98(7):1322-30.

19. Más Lago P, Ferrer H, Goyenechea A, Galindo A, Sarmiento L, Fonseca M, et al. Casos de poliomielitis paralítica asociada a la vacuna oral antipoliomielítica en Cuba (1963-2006). Rev Cubana Hig Epidemiol. 2008;46:1-14.

20. Kohler KA, Banerjee K, Hlady WG, Andrus JK, Sutter RW. Vaccine-associated paralytic poliomyelitis in India during 1999: decreased risk despite massive use of oral polio vaccine. Bull World Health Organ. 2002;80(3):210-6.

21. Murray CJL, López AD, eds. The global burden of disease: a comprehensive assessment of mortality and disability from diseases, injuries, and risk factors in 1990 and projected to 2020. Boston, MA: Harvard School of Public Health; 1996.

22. World Health Organization. Macroeconomics and health: investing in health for economic development. Report of the Commission on Macroeconomics and Health. Geneva: WHO; 2001. Hallado en http://whqlibdoc.who.int/ publications/2001/924154550x.pdf. Acceso el 7 de marzo de 2010.

23. Minor PD, Muir P. Enteroviruses. In: Zuckerman AJ, Banatvala JE, Pattison JR, Griffiths PD, Schoub BD, eds. Principles and practice of clinical virology. 5th ed. Chichester, UK: John Wiley \& Sons; 2004. Pp. 485-6.

24. Mahadevan $S$, Ananthakrishnan $S$, Srinivasan S, Nalini P, Puri RK, Badrinath S, et al. Poliomyelitis: 20 years-the Pondicherry experience. J Trop Med Hyg. 1989;92(6):416-21.

25. Andrus JK, Strebel PM, de Quadros CA, Olive JM. Risk of vaccine-associated paralytic poliomyelitis in Latin America, 1989-91. Bull World Health Organ. 1995;73(1):33-40.

26. De Oliveira LH, Struchiner CJ. Vaccineassociated paralytic poliomyelitis in Brazil, 1989-1995. Rev Panam Salud Publica. 2000; 7(4):219-24.

27. Teixeira-Rocha ES, Carmo EH, Tavares-Neto $\mathrm{J}$. The occurrence of vaccine-associated paralytic poliomyelitis in Brazil, 1995 to 2001. Rev Panam Salud Publica. 2005;18(1):21-4.

28. Nkowane BM, Wassilak SG, Orenstein WA, Bart KJ, Schonberger LB, Hinman AR, et al. Vaccine-associated paralytic poliomyelitis. United States: 1973 through 1984. J Am Med Assoc. 1987;257(10):1335-40.

29. Strebel PM, Sutter RW, Cochi SL, Biellik RJ, Brink EW, Kew OM, et al. Epidemiology of poliomyelitis in the United States one decade after the last reported case of indigenous wild virus-associated disease. Clin Infect Dis. 1992; 14(2):568-79.

30. Joce R, Wood D, Brown D, Begg N. Paralytic poliomyelitis in England and Wales, 1985-91. Br Med J. 1992;305(6845):79-82.

31. Griffiths UK, Botham L, Schoub BD. The costeffectiveness of alternative polio immunization policies in South Africa. Vaccine. 2006; 24(29-30):5670-8.

32. Tucker AW, Isaacs D, Burgess M. Costeffectiveness analysis of changing from live oral poliovirus vaccine to inactivated poliovirus vaccine in Australia. Aust N Z J Public Health. 2001;25(5):411-6.

33. Organización Panamericana de la Salud. Vigilancia de parálisis flácida aguda. Bol Sem Polio. 2006;21(48):2.

34. Grupo de Vigilancia de Inmunoprevenibles. Informe de la vigilancia de eventos de interés en salud pública: inmunoprevenibles. Santa Fe de Bogotá: Instituto Nacional de Salud; 2007. Hallado en http://www.ins.gov.co/ ?idcategoria $=5656 \#$. Acceso el 7 de marzo de 2010.

35. Prevots DR, Burr RK, Sutter RW, Murphy TV. Poliomyelitis prevention in the United States. Updated recommendations of the Advisory Committee on Immunization Practices (ACIP). Morb Mortal Wkly Rep. 2000;49(RR-5):1-22.

36. Le Saux N, Barrowman NJ, Moore DL, Whiting S, Scheifele D, Halperin S. Decrease in hospital admissions for febrile seizures and reports of hypotonic-hyporesponsive episodes presenting to hospital emergency departments since switching to acellular pertussis vaccine in Canada: a report from IMPACT. Pediatrics. 2003;112(5):e348.

37. Rennels MB, Englund JA, Bernstein DI, Losonsky GA, Anderson EL, Pichichero ME, et al. Diminution of the anti-polyribosylribitol phosphate response to a combined diphtheriatetanus-acellular pertussis/Haemophilus influenzae type $\mathrm{b}$ vaccine by concurrent inactivated poliovirus vaccination. Pediatr Infect Dis J. 2000;19(5):417-23.

38. Ekwueme DU, Strebel PM, Hadler SC, Meltzer MI, Allen JW, Livengood JR. Economic evaluation of use of diphtheria, tetanus, and acellular pertussis vaccine or diphtheria, tetanus, and whole-cell pertussis vaccine in the United States, 1997. Arch Pediatr Adolesc Med. 2000;154(8):797-803.

Manuscrito recibido el 18 de septiembre de 2009. Aceptado para publicación, tras revisión, el 2 de marzo de 2010. 
ABSTRACT Objective. Evaluate the cost-effectiveness of introducing the injectable inactivated polio vaccine (IPV) in Colombia versus the current system based on the use of the oral vaccine $(\mathrm{OPV})$.

Economic impact of introducing the injectable inactivated polio vaccine in Colombia
Methods. A Markov model was designed, based on a hypothetical cohort of newborns that would receive the IPV or the OPV vaccine, with a two-year follow-up and monthly estimates of the number of cases of vaccine-associated paralytic poliomyelitis (VAPP) that would emerge. The cost was analyzed from the perspective of the insurer (costs throughout life) and society (cases of VAPP and disability-adjusted life years [DALYs] prevented).

Results. From 1988 to 1998, some 22.5 million doses of OVP were administered in Colombia and nine cases of VAPP were detected, for a rate of $4.0 \times 10^{-7}$ dose. According to the model, 2 to 4 cases of VAPP could be anticipated in the following two years. The cost of treating the VAPP cases would total US\$302 008, with the cost of vaccination with OPV coming to US\$737 037 and with IPV, US\$5 527777 . Vaccination with IPV would prevent 64 DALYs, at a cost of US\$71 062 per DALY prevented; preventing one case of VAPP by substituting OPV with IPV would cost between US\$1.8 and US $\$ 2.2$ million.

Conclusions. Substituting OPV with IPV is not a cost-effective measure in Colombia, even if the cellular vaccine against whooping cough currently in use were replaced with an acellular vaccine combined with an IPV.

Key words Poliovirus vaccine, oral; poliovirus vaccine, inactivated; cost-benefit analysis; disabilityadjusted life years; mass vaccination; Colombia. 